\title{
Incentive contrast following repeated shifts in magnitude of food reward in the Skinner box
}

\author{
MITRI E. SHANAB, JEFF KONG, and JULIA DOMINO \\ California State University, Fresno, California 93740
}

\begin{abstract}
Thirty-two rats were trained in the Skinner box and received either large or small food reward in Phase 1. In Phase 2, half of each group was shifted to the other reward condition while the other half continued to receive the same reward magnitude. In Phase 3, Phase 1 conditions were reinstated for the shifted groups. Neither a positive contrast effect (PCE) nor a negative contrast effect (NCE) was obtained in Phase 2. Following a repeated shift in Phase 3, a strong NCE was obtained, but only a graphical, albeit statistically nonsignificant, PCE was observed. The results were discussed in terms of the older relative theories of Crespi and Helson as well as the more recent relativistic accounts of Amsel and Capaldi.
\end{abstract}

It has consistently been found in runway studies that subjects shifted from a large to a smaller food reward run significantly slower than control subjects receiving small reward all the time, thus reflecting a negative contrast effect (NCE). The evidence for the opposite phenomenon, namely for a positive contrast effect (PCE) in the runway is virtually nonexistent (Dunham, 1968). However, with appropriate controls for the assumed ceiling effects inherent in the running response, numerous studies have recently reported significant PCE based on shifts in magnitude of food reward (cf. Mellgren, 1972; Shanab, Birnbaum, \& Cavallaro, 1974; Shanab, Sanders, \& Premack, 1969). In the studies just cited, positive contrast was studied as a function of a single shift in reward magnitude. In other words, subjects were shifted from a small to a larger reward only once. In a repeated-shift design, Capaldi and Lynch (1967) reported no PCE regardless of whether their subjects were shifted once or twice from a small to a larger reward magnitude. These results are inconsistent with either the earlier findings of PCE (Crespi, 1942) or the more recent findings of PCE based on repeated shifts (Benefield, Oscos, \& Ehrenfreund, 1974; Shanab \& Ferrell, 1975; Shanab, Young, \& France, 1975). More recently, Shanab, France, and Young (1976), using sucrose reward, reported significant PCE and NCE following a repeated-shift procedure similar to that used by Capaldi and Lynch (1967). However, no NCE in the runway has been obtained based on single downshifts in either magnitude or concentration of liquid sucrose, or both (Barnes \& Tombaugh, 1973; Flaherty, Riley, \& Spear, 1973; Goodrich, 1962; Homzie \& Ross, 1962; Ison \& Rosen, 1968; Rosen, 1966; Rosen \& Ison, 1965; Shanab, Young, \& France, 1975). But significant NCE with sucrose was obtained in the licking response (Vogel, Mikulka, \& Spear, 1968), and the

Reprint requests should be sent to Mitri E. Shanab, Department of Psychology, California State University, Fresno, California 93740. barpress response (Collier \& Marx, 1959; Weinstein, 1970a, b).

The present study was designed to replicate and extend previous runway findings of single and repeated shifts in magnitude of food reward by using a different apparatus, namely a Skinner box, requiring a different response. Until now, all incentive contrast studies based on shifts in magnitude of food reward have been conducted in the runway with speed rather than rate of the response as the dependent variable. Based on the more recent findings of PCE (cf. Benefield et al., 1974; Shanab \& Ferrell, 1975; Shanab et al., 1976), it was hypothesized that PCE would occur in subjects shifted from small to large reward only if these subjects had previous experience with large reward, and that NCE would occur in subjects shifted from large to small reward only if they had prior commerce with small reward.

\section{METHOD}

\section{Design}

A three-phase experiment was run. In Phase 1, two groups of rats received either six $45-\mathrm{mg}$ Noyes pellets or one such pellet. In Phase 2, half of each group was shifted to the other reward condition while the other half continued to receive the same reward magnitude. In Phase 3, Phase 1 conditions were reinstated for the shifted groups. The rats were reinforced on a FI 30-sec schedule throughout the experiment.

\section{Subjects}

Thirty-two male albino rats of the Sprague-Dawley strain, approximately 90 days old at the beginning of the experiment, were used. The subjects were maintained at $80 \%$ of their freefeeding weight.

\section{Apparatus}

Two identical Lehigh Valley Electronics chambers controlled by standard solid state programming equipment were used. Appropriate modifications were made so that the feeder in each chamber could deliver either one $45-\mathrm{mg}$ food pellet or six such pellets. Each chamber was placed in a soundproof box equipped with an exhaust fan which served to mask external noises.

\section{Procedure}

Upon arrival from the supplier, the subjects were placed on 
free feeding for 2 weeks. For the following 2 weeks, the subjects were placed on a 24-h food deprivation schedule. During this period they were handled and allowed to eat five $45-\mathrm{mg}$ Noyes food pellets from the lid of a baby food jar. Following this handling period, each subject was allowed to explore its appropriate chamber for $5 \mathrm{~min} /$ day for 3 days. During this period, five food pellets were placed in the foodcup, and the equipment was turned on to adapt the subjects to the various noises. The subjects were randomly assigned to the two chambers.

Following magazine training, all subjects received continuous reinforcement training (CRF) for five sessions with 50 one-pellet reinforcements per session. Then the subjects were matched on the basis of their response rates over the last three sessions of their CRF training and randomly assigned to two groups: large or small rewarded groups. During the next two sessions, both groups were reinforced on a FI 15-sec schedule, the largerewarded groups (L) received six pellets, while the smallrewarded group (S) received one pellet for a total of six reinforcements.

Phase 1 of the experiment began when subjects were reinforced on a FI 30-sec schedule. Each daily session consisted of six 30 -sec cycles which were separated by 20 -sec time-out periods during which the houselight and cue lights over the foodcup were off and the response counter was inoperative. Thus each session comprised six discrete reinforcement periods. Phase 1 lasted 15 sessions.

Following stable performance, the subjects in each group were matched on the basis of their overall response rate over the last three sessions of Phase 1 and divided into four random groups. Half of the small-rewarded subjects were shifted to large reward (S-L), and the other half continued to receive the same small reward as before (S-S). Similarly, half of the largerewarded subjects continued to receive large reward (L-L), while the other half was shifted to small reward (L-S). Phase 2, which represented a 2 by 2 design, consisted of 15 sessions. In Phase 3, which also lasted for 15 sessions, Phase 1 conditions were reinstated for the shifted groups, yielding four random groups: LLL, SSS, LSL, SLS.

\section{RESULTS}

All analyses are based on overall response rate during each 3-min session. An analysis of variance over the last three sessions of Phase 1 revealed no significant magnitude effect $[F(1,30)<1]$. Since the results of a simple two-way analysis of variance test performed over the last 10 as well as the last 3 blocks of Phase 2 yielded highly similar results, only the former results will be reported. Neither Phase 1 conditions $[F(1,28)=1.73$, $\mathrm{p}>.05]$ nor the interaction of Phase 1 conditions with Phase 2 conditions $[\mathrm{F}(1,28)<1]$ was significant. However, Phase 2 conditions were significant $[F(1,28)$ $=5.72, \mathrm{p}<.05]$, indicating that large-rewarded subjects barpressed significantly faster than small-rewarded subjects. However, individual comparisons for PCE and NCE revealed nonsignificant results $[\mathrm{F}(1,28)<1$ for the difference between Groups L-L and S-L, and $F(1,28)$ $=1.50, \mathrm{p}>.05$ for the difference between Groups S-S and L-S].

As Figure 1 shows, there was more separation among the groups in Phase 3 than in the preceding phase. Rather rapid increases and decreases in response rate occurred in the shifted groups. Graphically, it appears that both positive and negative contrast effects were obtained. An analysis of variance with repeated measures over the last seven sessions yielded a highly significant Treatments effect $[F(3,28)=15.01, p<.001]$. Neither the Sessions effect nor the Treatments by Sessions effect was significant ( $F<1$ in both cases). The latter finding indicates that all four groups had reached a stable level of performance during the last seven sessions of Phase 3. Individual comparisons showed a significant NCE reflected by the difference between Groups SLS and $\operatorname{SSS}[F(1,28)=5.81, p<.05]$. However, the difference between Groups LSL and LLL failed to yield a significant PCE $[F(1,28)=1.75, p>.05]$.

\section{DISCUSSION}

The present study has revealed several interesting findings. No incentive contrast was obtained in the Skinner box based on either a single upward or downward shift. The absence of PCE is consistent with the numerous runway studies (betweensubjects designs) in which either food or sucrose was shifted (Dunham, 1968). The lack of PCE in this study is also consistent with the similar finding of an absence of positive behavioral contrast when rats rather than pigeons were used in singlesubject designs (cf. Freeman, 1971; Pear \& Wilkie, 1971; Shanab \& Gersh, 1976).

The absence of a NCE based on a single downshift in mag-

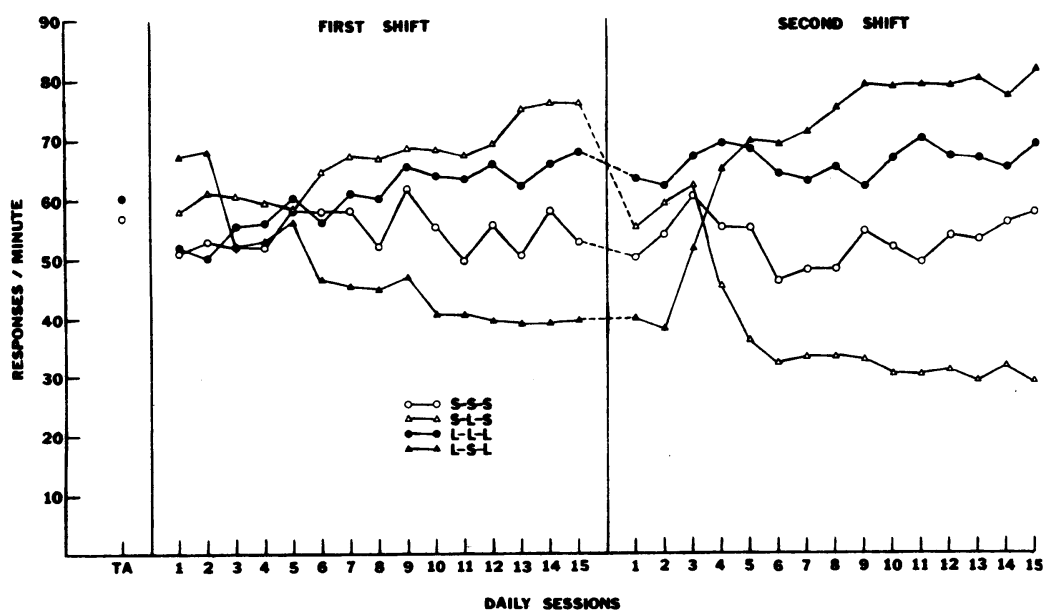

Figure 1. Mean responses per minute as a function of a single shift as well as double shift in magnitude of food reward. 
nitude of food reward is inconsistent with the findings of many runway studies (Dunham, 1968) but agrees with the results of many runway studies in which sucrose reward was downshifted (cf. Shanab, Young, \& France 1975) as well as with the infrequent occurrence of negative behavioral contrast in singlesubjects designs (Dunham, 1968).

The significant NCE obtained in Phase 3 is consistent with the similar finding based on shifts in sucrose solution (Shanab et al., 1976). The absence of NCE following a single downward shift in Phase 1, as well as the occurrence of NCE following a repeated shift in Phase 3, confirm one of the hypotheses tested, namely, that NCE would occur only if subjects shifted from large to small reward had previous experience with small reward. The similar finding of no PCE following a single upward shift and a graphical, albeit statistically nonsignificant, PCE following a repeated shift provides at least partial support to the other hypothesis.

The lack of statistical significance in spite of a clear graphical PCE is surprising, especially since seven of the eight upshifted rats showed extremely elevated response rates in Phase 3 compared to Phase 1 baseline rates. A closer examination of their data revealed that these subjects were probably responding very close to their physiological limit. For example, in terms of mean number of responses per minute over the last three sessions of Phase 3, the upshifted rats responded at the following high rates: 90 (70), $80(50), 85(45), 120(80), 80(70), 95(70), 80$ $(60)$, and 35 (35); the figures in parentheses refer to each subject's baseline in Phase 1 . The likelihood of a ceiling effect could be tested by using a VI instead of a discrete-trial FI schedule, or some other control technique for ceiling effects (cf. Shanab, Birnbaum, \& Cavallaro, 1974).

The lack of NCE following the first shift (Phase 2) does not agree with either the frustration (Amsel, 1962) or the generalization decrement hypothesis (Capaldi, 1967). According to these hypotheses, a downshift in reward magnitude should produce either frustration or a marked change in stimulus conditions so as to produce a significant decrement in the performance of the downshifted subjects relative to their nonshifted controls. Capaldi and Lynch (1967) reported results that presumably support this notion. They obtained a NCE (but not a PCE) following a single shift. However, a closer reading of their published article reveals that NCE was obtained on only one out of the 15 shifted trials. Thus the present lack of both PCE and NCE in Phase 2 is quite consistent with that obtained by Capaldi and Lynch (1967) in the runway. However, the finding of a NCE in this study following a second shift runs counter to that reported by Capaldi and Lynch (1967). According to these investigators, no NCE should be obtained if subjects have already been conditioned with small reward. This is because a shift to small reward after some experience with large reward would not produce any generalization decrement. It is conceivable that the discrepancy in findings could be attributed to the different procedures used in both studies. In the present study, response rate, massed trials, and prolonged training trials were used. In the Capaldi and Lynch study (1967), response speed, spaced (24-h ITI) trials, and rather short training trials (15 trials/phase) were used.

The significant NCE obtained in this study can be explained by either the frustration or generalization-decrement hypothesis by assuming that a second shift enhances the discriminability of the shifted conditions, thus allowing subjects to experience either frustration or generalization decrement, and hence show NCE. Capaldi (1974) has recently proposed a relativistic account of contrast which is reminiscent of other relative views of contrast (cf. Crespi, 1944; Helson, 1964). According to Capaldi (1974), a PCE would occur if expected reward is below obtained reward, while NCE would be predicted if expected reward is greater than obtained reward. Crespi (1944) earlier proposed that a PCE takes place whenever level of achievement exceeds level of expectation, and the opposite would be predicted for NCE. According to Helson (1964), PCE occurs whenever obtained reward exceeds that expected on the basis of a norm (adaptation level) formed by the subject as a result of experience with past reward conditions. While relative theories can explain contrast phenomena after these effects have occurred, they do not predict precisely when contrast would occur since expectancy, which is the focal concept in these theories, is only vaguely defined. Expectancy should be operationally defined so that it may be possible to deduce hypotheses to disconfirm the parent theories. However, the inconsistency in contrast findings suggests that until more empirical work has been done, it is probably premature to press for more rigorous theories.

\section{REFERENCES}

AMSEL, A. Frustrative nonreward in partial reinforcement and discrimination learning: Some recent history and a theoretical extension. Psychological Review, 1962, 69, 306-328.

Barnes, W., \& Tombaugh, T. N. Another failure to obtain negative incentive contrast following reductions in sucrose reward. Psychological Reports, 1973, 33, 801-802.

Benefield, R., Oscos, A., \& Ehrenfreund, D. Role of frustration in successive positive contrast. Journal of Comparative and Physiological Psychology, 1974, 86, 648-651.

Capaldi, E. J. A sequential hypothesis of instrumeñtal learning. In K. S. Spence \& J. T. Spence (Eds.) The psychology of learning and motivation (Vol. 1). New York: Academic Press, 1967. Pp. 67-156.

CaPaldi, E. J. Partial reward either following or preceeding consistent reward: A case of reinforcement level. Journal of Experimental Psychology, 1974, 102, 954-962.

CAPAldi, E. J., \& LYNCH, D. Repeated shifts in reward magnitude: Evidence in favor of an associational and absolute (noncontextual) interpretation. Journal of Experimental Psychology, 1967, 75, 226-235.

Collier, G., \& Marx, M. H. Changes in performance as a function of shifts in the magnitude of reinforcement. Journal of Experimental Psychology, 1959, 57, 305-309.

CRESPI, L. P. Quantitative variations of incentive and performance in the white rat. American Journal of Psychology, 1942, 55, 467-517.

Creśri, L. P. Amount of reinforcement and level of performance. Psychological Review, 1944, 51, 305-309.

Dunham, P. J. Contrasted conditions of reinforcement: A selective critique. Psychological Bulletin, 1968, 69, 295-315.

Flaherty, C. F., Riley, E. P., \& Spear, N. E. Effects of sucrose concentration and goal units on running behavior in the rat. Learning and Motivation, 1973, 4, 163-175.

Freeman, B. J. Behavioral contrast: Reinforcement frequency or response suppression? Psychological Bulletin, 1971, 75, 347-356.

GoODRICH, K. P. Supplementary report: Running speed as a function of sucrose concentration in a prior free drinking period. Psychological Reports, 1962, 11, 528-530.

Helson, H. Adaptation level theory. New York: Harper \& Row, 1964.

Homzie, M. J., \& Ross, L. E. Runway performance following a reduction in the concentration of a liquid reward. Journal of Comparative and Physiological Psychology, 1962, 55, 1029-1033.

Ison, J. R., \& Rosen, A. J. Extinction and reacquisition performance as a function of sucrose-solution rewards and numbers of acquisition trials. Psychological Reports, 1968, 22, 375-379.

Mellaren, R. L. Positive and negative contrast effects using delayed reinforcement. Learning and Motivation, 1972, 3, 185-193.

PEAR, J. J., \& WILkIE, D. M. Contrast and induction in rats on multiple schedules. Journal of the Experimental Analysis of Behavior, 1971, 15, 289-296.

Rosen, A. J. Incentive-shift performance as a function of 
magnitude and number of sucrose rewards. Journal of Comparative and Physiological Psychology, 1966, 62, 487-490.

Rosen, A. J., \& Ison, J. R. Runway performance following changes in sucrose rewards. Psychonomic Science, 1965, 2. $335-336$

Shanab, M. E., Birnbaum, D. W., \& Cavallaro, G. Positive contrast obtained in reacquisition following interpolation of nonreinforced or partially reinforced trials. Learning and Motivation, 1974, 5, 258-271.

Shanab, M. E., \& Ferrell, H. J. Transfer between downshift in reward magnitude and continuous delay of reward. Learning and Motivation, 1975, 6, 241-252.

Shanab, M. E., France, J., \& Young, T. Negative contrast effect obtained with downshifts in magnitude but not concentration of solid sucrose reward. Bulletin of the Psychonomic Society, 1975, 5, 429-432.

Shanab, M. E., France, J., \& Young, T. Positive and negative contrast effects obtained following shifts in liquid sucrose reward in thirsty rats. Animal Learning \& Behavior, 1976, 4, 9-12.
Shanab, M. E., \& Gersh, W. D. Behavioral contrast as a function of two different reinforcers. Journal of General Psychology, 1976, 94, 29-45.

Shanab, M. E., Sanders, R., \& Premack, D. Positive contrast in the runway obtained with delay of reward. Science, 1969, 164, 724-725.

Shanab, M. E., Young, T., \& France, J. Negative contrast as a function of downshifts in magnitude of sucrose concentrations in thirsty rats. Bulletin of the Psychonomic Society, 1975, 5, 381-384.

Vogel, J. R., Mikulka, R. J., \& Spear, N. E. Effects of shifts in sucrose and saccharine concentrations on licking behavior in the rat. Journal of Comparative and Physiological Psychology, 1968, 66, 661-666.

Weinstein, L. Negative incentive contrast with sucrose. Psychonomic Science, 1970, 19, 13-14. (a)

Weinstein, L. Negative incentive contrast effects with saccharine vs. sucrose and partial reinforcement. Psychonomic Science, 1970, 21, 276-278. (b)

(Received for publication June 14, 1976.) 